\title{
Highlights from the LHCb experiment
}

\author{
Katharina Müller, on behalf of the LHCb Collaboration* \\ University of Zurich \\ E-mail: kmueller@physik.uzh.ch
}

\begin{abstract}
A selection of the most recent and relevant measurements by the LHCb experiment is presented. The large samples of charmed hadron decays yield some of the world's most sensitive searches for direct and indirect $C P$ violation in the charm sector and allowed for the first observation of $C P$ violation in charm. The results from $B$-decays include measurements with unprecedented precision of $C P$ violating parameters, a recent update of the LHCb combination of the CKM angle $\gamma$ as well as test of lepton flavour universality and searches for lepton flavour violating decays. Two new LHCb spectroscopy results are shown: The observation of new resonances in the $\Lambda_{b}^{0} \pi^{+} \pi^{-}$spectrum and the observation of new pentaquark states. Finally, new results of $b$ hadron production in proton-lead collisions and charm production in fixed target collisions are presented.
\end{abstract}

European Physical Society Conference on High Energy Physics - EPS-HEP2019 -

10-17 July, 2019

Ghent, Belgium

${ }^{*}$ Speaker. 


\section{Introduction}

The LHCb detector is a forward spectrometer designed for precision studies of $b$ - and $c$-hadron decays[1]. Of particular relevance for the measurements discussed in these proceedings is its excellent precision in reconstructing vertices associated to primary $p p$ interactions and to the decays of long-lived particles such as $B$ and $D$ mesons and $\tau$ leptons. The momenta of charged particles are reconstructed with high precision by a system of tracking detectors. Different types of charged hadrons, such as protons, kaons and pions, are distinguished using information from two ring-imaging Cherenkov detectors, while photons, electrons and muons are identified by means of a calorimeter system and a muon detector. The measurements presented in the following rely on a data sample of $p p$ collisions at centre-of-mass energies, $\sqrt{s}$, of 7 and $8 \mathrm{TeV}$ collected by the LHCb detector during Run 1 and corresponding to an integrated luminosity of $3 \mathrm{fb}^{-1}$ and up to $6 \mathrm{fb}^{-1}$ collected at $\sqrt{s}=13 \mathrm{TeV}$ in Run 2 as well on samples of proton lead and fixed target collisions collected in special runs.

\section{Charm physics}

This section presents the first observation of $C P$ violation in charm and some of the world's most sensitive searches for direct and indirect $C P$ violation in the charm sector.

\subsection{Observation of $C P$ violation in charm}

$C P$ violation $(C P V)$, is a necessary condition for the dynamical generation of the baryon asymmetry of the universe. The Standard Model of particle physics (SM) includes $C P V$ through an irreducible complex phase in the Cabibbo-Kobayashi-Maskawa (CKM) quark-mixing matrix. The realisation of $C P V$ in weak interactions has been established in the $K$ - and $B$-meson systems by several experiments with all the results in agreement with the CKM formalism. However, the size of $C P V$ in the SM appears to be too small to account for the observed matter-antimatter asymmetry, suggesting the existence of sources of $C P V$ beyond the SM.

While $C P V$ in the beauty sector is well established the observation of $C P V$ in the charm sector had not been achieved, despite decades of experimental searches. Charm hadrons provide a unique opportunity to measure $C P V$ with particles containing only up-type quarks. The size of $C P V$ in charm decays is expected to be very small in the SM, with asymmetries typically of the order of $10^{-4}-10^{-3}$. The uncertainties however are large due to the presence of low-energy stronginteraction effects.

The LHCb collaboration performed a search for $C P V$ in $D^{0} \rightarrow K^{-} K^{+}$and $D^{0} \rightarrow \pi^{-} \pi^{+}$decays $^{1}$, using the full Run 2 dataset with $6 \mathrm{fb}^{-1}$ collected at $\sqrt{s}=13 \mathrm{TeV}$ [2]. The time-dependent $C P$ asymmetry, $A_{C P}(f ; t)$, between states produced as $D^{0}$ or $\bar{D}^{0}$ decaying to a $C P$ eigenstate $f$ at time $t$ is defined as $A_{C P}(f ; t)=\left(\Gamma\left(D^{0}(t) \rightarrow f\right)-\Gamma\left(\bar{D}^{0}(t) \rightarrow f\right)\right) /\left(\Gamma\left(D^{0}(t) \rightarrow f\right)+\Gamma\left(\bar{D}^{0}(t) \rightarrow f\right)\right)$ where $\Gamma$ denotes the time-dependent rate of a given decay and $f=K^{+} K^{-}$or $f=\pi^{+} \pi^{-} . A_{C P}$ receives contributions from direct $C P V\left(a_{C P}^{\text {dir }}\right)$ as well as indirect $C P V\left(a_{C P}^{i n d i r}\right)$ in the mixing and the interference between mixing and decay. Experimentally, it is easier to determine $\triangle A_{C P}$, the difference

\footnotetext{
${ }^{1}$ The inclusion of charge-conjugate decay modes is implied throghout these proceedings.
} 
in
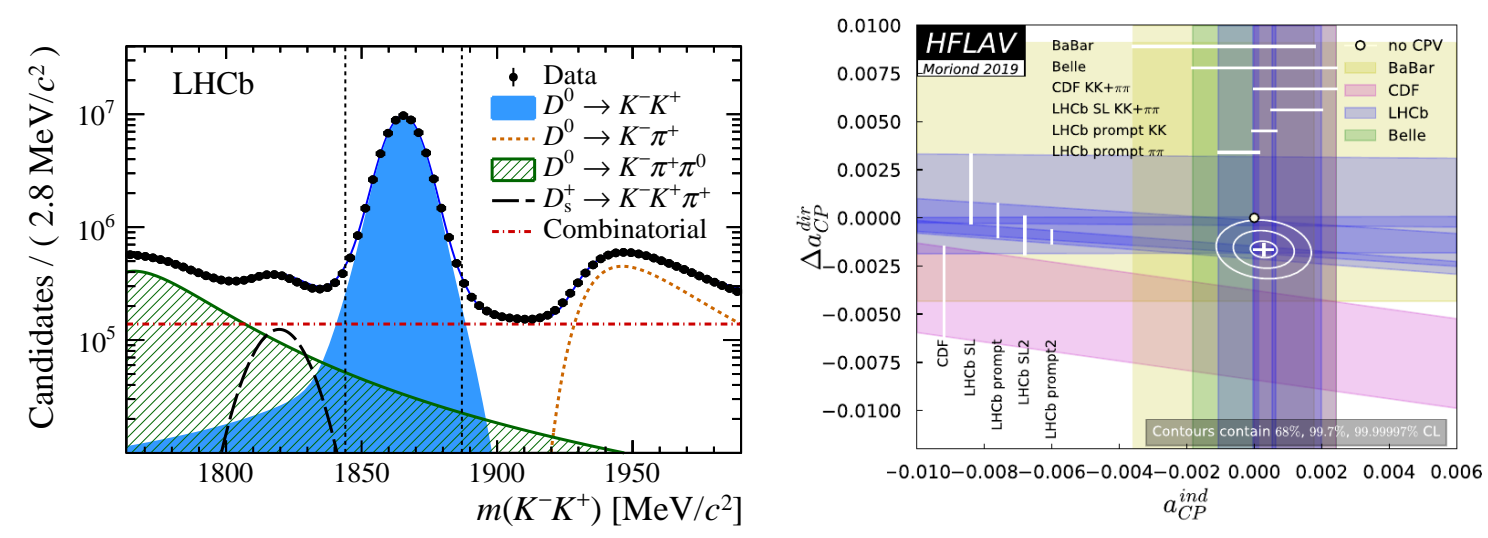

Figure 1: Left: Invariant-mass distributions of $D^{0} \rightarrow K^{-} K^{+}$candidates in the prompt sample with the fit results overlaid. The various components included in the fit model are indicated in the legend (from [2]). Right: Indirect vs direct $C P$ parameters from independent experimental measurements, together with the world average denoted as white cross. The white dot corresponds to the no $C P V$ hypothesis (Figure from [3]).

$C P$ asymmetries between $D^{0} \rightarrow K^{-} K^{+}$and $D^{0} \rightarrow \pi^{-} \pi^{+}$decays, where many systematic uncertainties cancel. In the limit of U-spin symmetry, $a_{C P}^{d i r}$ is equal in magnitude and opposite in sign for $K^{-} K^{+}$and $\pi^{-} \pi^{+}$and $\Delta A_{C P}=A_{C P}\left(K^{-} K^{+}\right)-A_{C P}\left(\pi^{-} \pi^{+}\right)$simplifies to $\Delta A_{C P} \simeq \Delta a_{C P}^{d i r}-$ $\Delta\langle t\rangle / \tau\left(D^{0}\right) A_{\Gamma}(f)$, where $\tau\left(D^{0}\right)$ is the $D^{0}$ lifetime and $A_{\Gamma}$ the asymmetry between the $D^{0}$ and $\bar{D}^{0}$ effective decay widths, which is assumed to be independent of the final state. The initial flavour of the $D$ meson is tagged by the charge of the pion in prompt decays $\left(D^{*+} \rightarrow D^{0} \pi^{+}\right)$, and by the muon charge in secondary production $\left(\bar{B}^{0} \rightarrow D^{0} \mu^{-} X\right)$, where $X$ stands for potential additional particles. The combination of the two tagging methods yields $\Delta A_{C P}^{\pi-t a g}=[-18.2 \pm 3.2 \pm 0.9] \times 10^{-4}$, $\Delta A_{C P}^{\mu-t a g}=[-9 \pm 8 \pm 5] \times 10^{-4}$, where the first uncertainty is statistical and the second systematical [2]; the combination with the Run 1 analyses [4] gives $\Delta A_{C P}=(-15.4 \pm 2.9) \times 10^{-4}$. The significance of the deviation from zero corresponds to 5.3 standard deviations and is the first observation of $C P V$ in the decay of charm hadrons. The result is consistent with, although at the upper end of the SM expectations. Figure 1 shows the invariant-mass distribution of $D^{0} \rightarrow K^{-} K^{+}$ candidates in the prompt sample with fit results overlaid (left) and a compilation of measurements of the indirect vs direct $C P$ parameters together with the world average (right).

\section{2 $\quad \mathrm{A}_{\Gamma}$ in $D^{0} \rightarrow K^{-} K^{+}$and $D^{0} \rightarrow \pi^{-} \pi^{+}$}

The $C P V$ parameter $A_{\Gamma}$ describes the asymmetries of the time-dependent decay rates of $D^{0}$ and $\bar{D}^{0}$ decays and is sensitive to $C P V$ in the mixing and in the interference between mixing and decay. Most recent SM calculations predict $A_{\Gamma} \simeq 3 \times 10^{-5}$ [5], one order of magnitude below the current experimental precision. Therefore, a value of the $A_{\Gamma}$ significantly different from zero would indicate the existence of new interactions beyond the SM. The value of $A_{\Gamma}$ is also a necessary ingredient 
for a precise determination determine of $C P$ asymmetries from the measurement of time-integrated asymmetries as in Sect. 2.1.

The LHCb collaboration performed a measurement of $A_{\Gamma}$ in $D^{0} \rightarrow K^{-} K^{+}$and $D^{0} \rightarrow \pi^{-} \pi^{+}$ decays, using a dataset of $1.9 \mathrm{fb}^{-1}$ collected at a $\sqrt{s}=13 \mathrm{TeV}$ [6]. The flavour of the $D^{0}$ meson is inferred from the charge of the pion in $D^{*+} \rightarrow D^{0} \pi^{+}$decays. The asymmetries of the timedependent decay rates of $D^{0}$ and $\bar{D}^{0}$ decays are measured to be $A_{\Gamma}=(1.3 \pm 3.5$ (stat $) \pm 0.7$ (syst) $) \times$ $10^{-4}$ for $D^{0} \rightarrow K^{+} K^{-}$and $(11.3 \pm 6.9$ (stat) \pm 0.8 (syst) $) \times 10^{-4}$ for $D^{0} \rightarrow \pi^{+} \pi^{-}$[6]. Combining the two channels and the previous LHCb [7] result the values for $A_{\Gamma}$ and the difference $\Delta A_{\Gamma}$ are $A_{\Gamma}=(0.9 \pm 2.1$ (stat) \pm 0.7 (syst) $) \times 10^{-4}$ and $\Delta A_{\Gamma} \equiv A_{\Gamma}\left(D^{0} \rightarrow K^{+} K^{-}\right)-A_{\Gamma}\left(D^{0} \rightarrow \pi^{+} \pi^{-}\right)=(0.9 \pm$ 2.1 (stat) \pm 0.7 (syst) $) \times 10^{-4}$, consistent with zero.

\subsection{Oscillations of charm mesons}

Oscillations of neutral charm mesons occur because their mass eigenstates are linear combinations of the flavor eigenstates: $\left|D_{1,2}\right\rangle \equiv p\left|D^{0}\right\rangle \pm q\left|D^{0}\right\rangle$, where $p$ and $q$ are complex parameters and $D_{1(2)}$ the $C P$-even (odd) state. In the limit of $C P$ symmetry the oscillation rate depends only on the mixing parameters $x \equiv\left(m_{1}-m_{2}\right) c^{2} / \Gamma$ and $y \equiv\left(\Gamma_{1}-\Gamma_{2}\right) /(2 \Gamma)$, where $m_{1(2)}$ and $\Gamma_{1(2)}$ are the mass and decay width of the $D_{1(2)}$, and $\Gamma=\left(\Gamma_{1}+\Gamma_{2}\right) / 2$. If $C P$ symmetry is violated, the oscillation rates for mesons produced as $D^{0}$ and $\bar{D}^{0}$ differ.

The LHCb collaboration measured charm oscillations in $D^{0} \rightarrow K_{S}^{0} \pi^{+} \pi^{-}$decays using the full Run 1 dataset with about 1.3 million prompt and 1 million secondary candidates [8]. The measurement is based on a model-independent approach (bin-flip method), which is optimized for the measurement of the parameter $x$ [9].

Allowing for $C P V$ in charm mixing, or in the interference between mixing and decay, the $C P$ averaged normalized mass difference $x_{C P}=[2.7 \pm 1.6$ (stat) \pm 0.4 (syst $\left.)\right] \times 10^{-3}$, the most precise determination by a single experiment, and the $C P$-violating parameter $\Delta x=[-0.53 \pm 0.70$ (stat) \pm 0.22 (syst) $] \times 10^{-3} .^{2}$ The new world average $x_{C P}=\left(3.9_{-1.2}^{+1.1}\right) \times 10^{-3}$ provides the first evidence for a mass difference between the neutral charm mesons. The impact of this measurement on global averages of the charm-mixing parameters is shown in Fig. 2 (left).

\section{Beauty physics}

The following results from $B$-decays at $\mathrm{LHCb}$ include measurements with unprecedented precision of $C P$ violating parameters, a recent update of the LHCb combination of the CKM angle $\gamma$ as well as test of lepton flavour universality and searches for lepton flavour violating decays.

\subsection{Measurement of the CKM angle $\gamma$}

The measurement of the angle $\gamma$ of the CKM unitarity triangle, $\arg \left(\left(V_{u d} V_{u b}^{*}\right) /\left(V_{c d} V_{c b}^{*}\right)\right)$, is considered as a SM benchmark: from a theoretical point of view it is very clean as it is dominated by tree level processes, where higher contributions from loops are very suppressed $\left(\mathrm{O}\left(10^{-4}\right)\right)$. It can be measured in tree level $B$-hadron decays exploiting the interference of $b \rightarrow c$ and $b \rightarrow u$ transitions. Unfortunately, the experimental determination of $\gamma$ is quite challenging since the visible branching

\footnotetext{
${ }^{2}$ Conservation of $C P$ symmetry in mixing, or in the interference between mixing and decay, implies $x_{C P}=x$.
} 

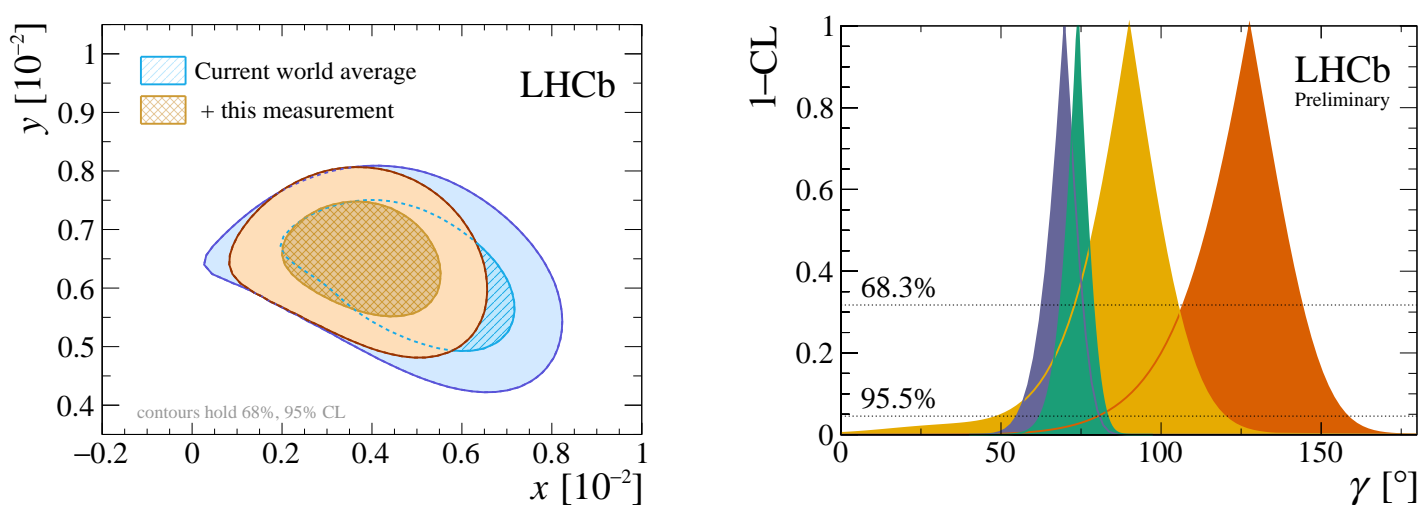

Figure 2: Left: Impact of the measurement of charm oscillations in $D^{0} \rightarrow K_{S}^{0} \pi^{+} \pi^{-}$decays on current global averages of charm-mixing parameters. The hatched and shaded areas in the bottom panels indicate the $68 \%$ and $95 \%$ confidence regions, respectively (from [8]). Right: 1-CL plots, using the profile likelihood method, for combinations of tree level measurements of $\gamma$ split by the initial $B$ meson flavour: (orange) $B_{s}^{0}$ initial states, (yellow) $B^{0}$ initial states, (blue) $B^{+}$initial states and (green) the full combination (from [10]).

fractions of the relevant $B$ decays and the typical size of these interferences are quite small. The best precision on the angle $\gamma$ results from a combination of all the possible measurements that exploit different analysis methods and external inputs, such as amplitude ratios and strong phases of the involved particle decays.

The LHCb collaboration performed a combination of tree-level measurements of the CKM angle $\gamma$ [10]. The results are obtained from time-integrated measurements of $B^{+} \rightarrow D K^{+}, B^{+} \rightarrow$ $D^{*} K^{+}, B^{+} \rightarrow D K^{+}, B^{0} \rightarrow D K^{0}, B^{0} \rightarrow D K^{+-}$and $B^{+} \rightarrow D K^{+} \pi^{+} \pi^{-}$decays and time-dependent analyses of $B_{s}^{0} \rightarrow D_{s} \mp K^{ \pm}$and $B^{0} \rightarrow D^{\mp} \pi^{ \pm}$decays. This combination includes new and updated results compared to the previous LHCb combination, giving a best fit value $\gamma=\left(74.0_{-5.8}^{+5.0}\right)^{\circ}$, where the uncertainty includes both statistical and systematic contributions and is the most precise determination of $\gamma$ from a single experiment to date [10]. The origin of the sensitivity to $\gamma$ of the various decay modes in the combination is shown in Fig. 2 (right) by performing profile likelihood scans using subsets of the input observables. The results from the different $B$ meson flavours are consistent at the $2 \sigma$ level. The result for $\gamma$ is in agreement with the combination from HFLAV $\gamma=\left(71.1_{-5.3}^{+4.6}\right)^{\circ}$ and the result from CKM fitter $\gamma=\left(65.8_{-1.7}^{+1.0}\right)^{\circ}$.

\subsection{The $B_{S}$ mixing phase $\phi_{s}$ from $B_{s} \rightarrow J / \psi K K$ and $B_{s} \rightarrow J / \psi \pi \pi$}

Measurements of $C P V$ in final states that can be populated both by direct decay and via mixing provide an excellent way of looking for physics beyond the SM as New Physics (NP) particles can enter in the loop and thus affect the relative $C P$ phase. Direct decays into non-flavour-specific final states can interfere with those that undergo $B_{s}^{0}-\bar{B}_{s}^{0}$ mixing before decaying. This interference can result in $C P V$ and manifests itself through a nonzero value of the phase $\phi_{s}=-\arg (\Lambda)$, where the parameter $\Lambda=\arg [(q / p)(\bar{A} / A)]$ describes $C P V$ in the interference between mixing and decay. Here, $A$ and $\bar{A}$ are the amplitudes for a $B_{s}^{0}$ or $\bar{B}_{s}^{0}$ meson to decay to the same final state and the complex parameters $p=\left\langle B_{s}^{0} \mid B_{L}\right\rangle$ and $q=\left\langle\bar{B}_{s}^{0} \mid B_{H}\right\rangle$ describe the relation between the flavour and the mass eigenstates (light, $L$, and heavy, $H$ ). In the absence of $C P V$ in the decay and assuming 

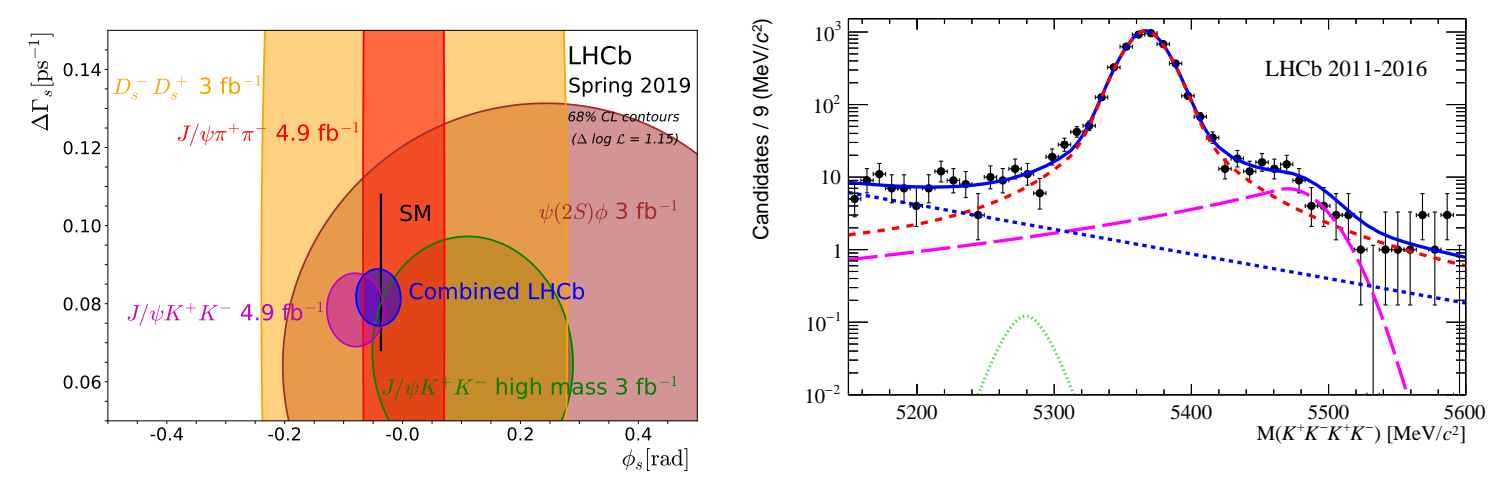

Figure 3: Left: Regions of $68 \%$ confidence level in the $\phi_{s}-\Delta \Gamma_{s}$ (the decay width difference of the $B_{s}^{0}$ mass eigenstates) plane for the individual LHCb measurements and a combined contour (in blue). The $B_{s}^{0} \rightarrow$ $J / \psi K^{+} K^{-}$[11] (magenta) and $B_{s}^{0} \rightarrow J / \psi \pi^{+} \pi^{-}$[12] (red) contours show the Run 1 and Run 2 combined numbers. The $\phi_{s}$ and $\Delta \Gamma_{s}$ predictions are indicated by the thin black rectangle. Right: Fit to the four-kaon invariant mass distributions for $B_{s}^{0} \rightarrow \Phi \Phi$ candidates. The total PDF is shown as a blue solid line, $B_{s}^{0} \rightarrow \Phi \Phi$ as a red dashed line, $B^{0} \rightarrow \Phi \Phi$ as a green dotted line, the $\Lambda_{b}^{0} \rightarrow \Phi p K$ contribution as a magenta long-dashed line and the combinatorial background as a blue short-dashed line (from [13]).

negligible $C P V$ in $B_{s}^{0}-\bar{B}_{s}^{0}$ oscillation, $|\Lambda|$ is expected to be unity while many NP models predict larger values for this phase if non-SM particles were to contribute to $B_{s}^{0}-\bar{B}_{s}^{0}$ oscillations.

The LHCb collaboration used $B_{s} \rightarrow J / \psi K K[11]$ and $B_{s} \rightarrow J / \psi \pi \pi[12]$ decays which have a clean signature and a high yield (about $117^{\prime} 000 B_{s} \rightarrow J / \psi K K$ and $33^{\prime} 500 B_{s} \rightarrow J / \psi \pi \pi$ candidates) using $2 \mathrm{fb}^{-1}$ of Run 2 . The flavour of the $B_{s}$ is determined from the decay of the other $b$ hadron in the event. Combined with the analysis of the Run 1 data this yields the most precise determination of $\phi_{s}=(-41 \pm 25) \mathrm{mrad}$. The value of $\phi_{s}$ is consistent with expectations assuming the SM and with no $C P V$ in the interference of $B_{s}^{0}-\bar{B}_{s}^{0}$ oscillation. Figure 3 (left) shows the individual and combined values of $\phi_{s}$ in the $\phi_{s}-\Delta \Gamma_{s}$ (the decay width difference of the $B_{s}^{0}$ mass eigenstates) plane.

\subsection{Measurement of $C P$ violation in $B_{s}^{0} \rightarrow \phi \phi$}

In the SM the $B_{s}^{0} \rightarrow \phi \phi$ decay is forbidden at tree level and proceeds predominantly via a gluonic $\bar{b} \rightarrow \bar{s} s \bar{s}$ loop process which makes this channel an excellent probe of new heavy particles entering the loop. The $C P$-violating phase, $\phi^{\bar{s} s \bar{s}}$ in the $B_{s}^{0} \rightarrow \phi \phi$ decay is expected to be small in the SM, recent calculations provide an upper limit of $0.02 \operatorname{rad}[14]$.

LHCb measured the time-dependent $C P$-violating asymmetry in $B_{s}^{0} \rightarrow \phi \phi$ decays in a sample corresponding to an integrated luminosity of $5.0 \mathrm{fb}^{-1}$ with a signal yield of around $9000 B_{s}^{0} \rightarrow \phi \phi$ candidates [13]. Their four-kaon invariant mass distribution is shown in Fig. 3 (right). The $C P$ violating phase $\Phi^{\bar{s} s \bar{s}}$ is measured to be $-0.073 \pm 0.115$ (stat) \pm 0.027 (syst) rad and the direct $C P$ violation parameter $\Lambda$ is found to be $|\Lambda|=0.99 \pm 0.05$ (stat) \pm 0.01 (syst), both values are determined in a helicity-independent manner. In addition, the most stringent limit on the branching fraction of the $B^{0} \rightarrow \phi \phi$ decay is measured to be $\mathscr{B}\left(B^{0} \rightarrow \phi \phi\right)<2.7 \times 10^{-8}$ at $90 \% \mathrm{CL}$. 

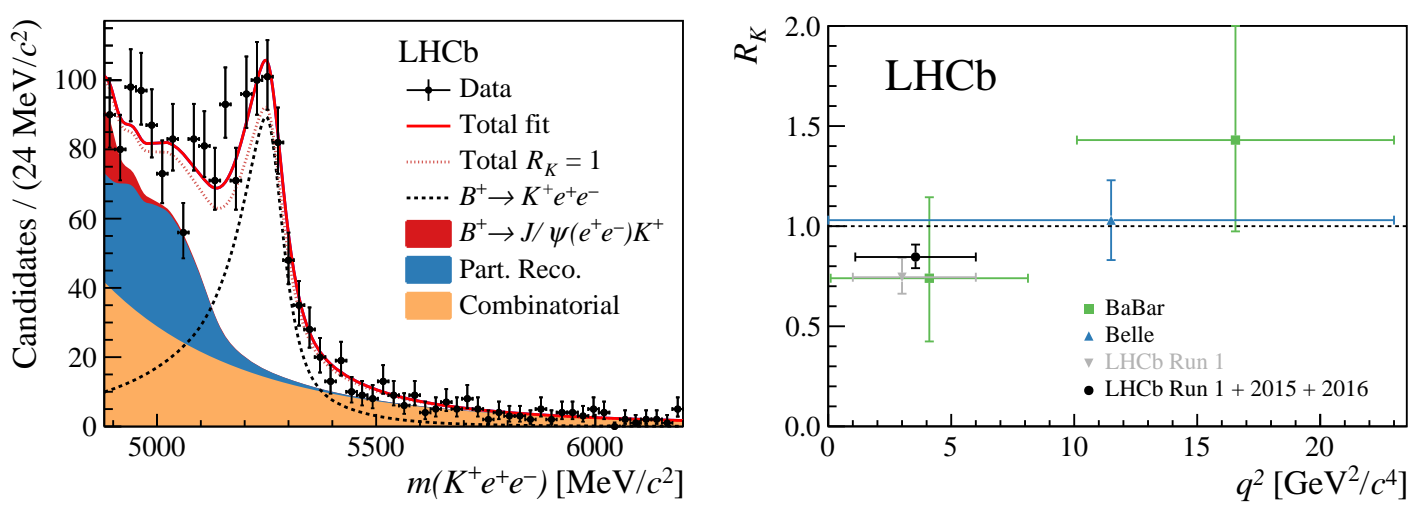

Figure 4: Left: Fit to the $m\left(K^{+} e^{+} e^{-}\right)$invariant mass distribution for non resonant decays of $B^{+} \rightarrow K^{+} e^{+} e^{-}$ (from [15]). Right: Comparison of the LHCb $R_{K}$ measurements with previous experimental results from $\mathrm{LHCb}$ and the B factories (from [15]).

\subsection{New measurement of $R_{K}$}

In the SM, the electroweak gauge bosons $Z$ and $W^{ \pm}$have identical couplings to all three lepton flavours. This means that branching fractions of decays involving different lepton families do not depend on lepton flavour but differ only by phase space and helicity-suppressed contributions. A very clean test for NP can hence be performed by taking ratios of $b \rightarrow s \ell^{+} \ell^{-}$decays to different lepton species. Since these decays are not allowed at tree level the branching fractions are highly sensitive to NP effects.

LHCb has recently published an update of the measurement of $R_{K}$, the ratio of branching fractions of the decays $B^{+} \rightarrow K^{+} \mu^{+} \mu^{-}$and $B^{+} \rightarrow K^{+} e^{+} e^{-}$, in the dilepton mass-squared region $1.1<q^{2}<6.0 \mathrm{GeV}^{2} / c^{4}$, using the full statistics of Run 1 and part of Run 2, corresponding to an integrated luminosity of $5 \mathrm{fb}^{-1}$ [15]. To cancel experimental uncertainties in the absolute efficiencies of the measurements, the ratio $R_{K}$ is measured as double ratio, normalising the non-resonant signal mode to the resonant $J / \psi$ mode:

$$
R_{K}=\frac{\mathscr{B}\left(B \rightarrow K \mu^{+} \mu^{-}\right)}{\mathscr{B}\left(B \rightarrow K J / \psi\left(\rightarrow \mu^{+} \mu^{-}\right)\right)} \times \frac{\mathscr{B}\left(B \rightarrow K J / \psi\left(\rightarrow e^{+} e^{-}\right)\right)}{\mathscr{B}\left(B \rightarrow K e^{+} e^{-}\right)} .
$$

The candidates for the normalisation channel $B \rightarrow K J / \psi\left(\rightarrow \ell^{+} \ell^{-}\right)$are selected using similar criteria to that of the non-resonant channel. The main experimental challenge of the measurement is the reconstruction of the electrons in the LHCb detector as the electrons are highly affected by bremsstrahlung. Signal yields are extracted using unbinned extended maximum likelihood fits to the reconstructed $B$ mass in the $J / \psi$ and the non-resonant channels; the fit to the non-resonant channel for the electron mode is shown in Fig. 4 (right).

The ratio of branching fractions is measured to be $R_{K}=0.846_{-0.054}^{+0.060}$ (stat $)_{-0.014}^{+0.016}$ (syst). This is the most precise measurement of $R_{K}$ to date and is compatible with the SM at the level of 2.5 standard deviations [15]. A comparison to other measurements is shown in Fig 4 (right).

\subsection{Search for Lepton flavour violating decays}

The observation of neutrino oscillations has provided the first evidence for lepton-flavour violation 
(LFV) in neutral leptons. In the charged sector LFV is negligible in the SM and any observation of a charged LFV decay would be a clear evidence for NP. LHCb recently performed searches for the lepton flavour violating decays $B_{s}^{0} \rightarrow \tau^{ \pm} \mu^{\mp}$ and $B^{0} \rightarrow \tau^{ \pm} \mu^{\mp}$ [16] and $B^{+} \rightarrow K^{+} \mu^{ \pm} e^{\mp}$ [17], both are based on the full Run 1 dataset with an integrated luminosity of $3 \mathrm{fb}^{-1}$.

In the SM the branching fraction for the decay $B_{s}^{0} \rightarrow \tau^{ \pm} \mu^{\mp}$ is of the order of $10^{-54}$ while NP models can increase it to $\mathscr{O}\left(10^{-8}-10^{-5}\right)$. The signal yield is evaluated by performing a simultaneous unbinned maximum-likelihood fit to the reconstructed $B$ invariant mass of $B_{(s)}^{0} \rightarrow$ $\tau^{ \pm} \mu^{\mp}\left(\tau^{-} \rightarrow \pi^{-} \pi^{+} \pi^{-} \nu_{\tau}\right)$ and four samples corresponding to different BDT bins that is used to separate signal from background. No significant signal is observed and upper limits are set on the branching fractions of $\mathscr{B}\left(B_{s}^{0} \rightarrow \tau^{ \pm} \mu^{\mp}\right)<4.2 \times 10^{-5}$ and $\mathscr{B}\left(B^{0} \rightarrow \tau^{ \pm} \mu^{\mp}\right)<1.4 \times 10^{-5}$ at 95\% confidence level. These are the first limit on $\mathscr{B}\left(B_{s}^{0} \rightarrow \tau^{ \pm} \mu^{\mp}\right)$ and the world's best limit on $\mathscr{B}\left(B^{0} \rightarrow \tau^{ \pm} \mu^{\mp}\right)[16]$.

NP models including leptoquarks, extended gauge bosons or $\mathrm{CP}$ violation in the neutrino sector predict branching fractions for the decay $B^{+} \rightarrow K^{+} \mu^{ \pm} e^{\mp}$ of the order $10^{-8}-10^{-10}$. No excess is observed over the background-only hypothesis, and the resulting upper limits on the branching fractions are $\mathscr{B}\left(B^{+} \rightarrow K^{+} \mu^{-} e^{+}\right)<9.5 \times 10^{-9}$ and $\mathscr{B}\left(B^{+} \rightarrow K^{+} \mu^{+} e^{-}\right)<8.8 \times 10^{-9}$ at $95 \%$ confidence level. The results improve the current best limits on the decays by more than one order of magnitude and impose strong constraints on NP models [17].

\section{Spectroscopy}

\subsection{New resonances in the $\Lambda_{b}^{0} \pi^{+} \pi^{-}$spectrum}

The LHCb collaboration studied the $\Lambda_{b}^{0} \pi^{+} \pi^{-}$spectrum in the extended mass region between 6.10 and $6.25 \mathrm{GeV}$ using the combined Run 1 and Run 2 dataset corresponding to an integrated luminosity of $9 \mathrm{fb}^{-1}$ [18]. There is a clear excess of $\Lambda_{b}^{0} \pi^{+} \pi^{-}$candidates around $6.15 \mathrm{GeV}$ over the background for both $\Lambda_{b}^{0}$ decay modes $\Lambda_{b}^{0} \rightarrow \Lambda_{c}^{+} \pi^{-}$and $\Lambda_{b}^{0} \rightarrow J / \psi p K^{-}$. An analysis of the mass spectra for the regions enriched by the $\Sigma_{b}^{(*)}$ as well as the non resonant region is shown in Fig. 5 (left) for $\Lambda_{b}^{0} \rightarrow \Lambda_{c}^{+} \pi^{-}$. The excess of $\Lambda_{b}^{0} \pi^{+} \pi^{-}$candidates can be described by two narrow mass states where the high mass state predominantly decays via intermediate $\Sigma_{b}^{*}$ and $\Sigma_{b}$ and the decay via $\Sigma_{b}$ is suppressed for the low mass state. The masses of the two states measured in this analysis are consistent with the expectation for a $\Lambda_{b}(1 \mathrm{D})$-doublet.

\subsection{Observation of new pentaquark states}

The field of exotic spectroscopy is extremely rich and productive. Since the observation of significant $J / \psi p$ pentaquark structures in $\Lambda_{b}^{0} \rightarrow J / \psi p K$ decays by LHCb [20] four years ago several observations and searches for pentaquark states have been performed. The new analysis of $\Lambda_{b}^{0} \rightarrow J / \psi p K$ decays by LHCb uses the full Run 1 and Run 2 dataset with a total integrated luminosity of $9 \mathrm{fb}^{-1}$ corresponding to a nine-fold increase in statistics and sheds more light onto the $J / \psi p$ structures [19]. Figure 5 (right) shows the fit to the $m_{J / \psi p}$ distribution. The previously reported $P_{c}(4450)^{+}$peak is confirmed and resolved at $5.4 \sigma$ significance into two narrow states: the $P_{c}(4440)^{+}$and $P_{c}(4457)^{+}$exotic baryons. A narrow companion state, $P_{c}(4312)^{+}$, is discovered 

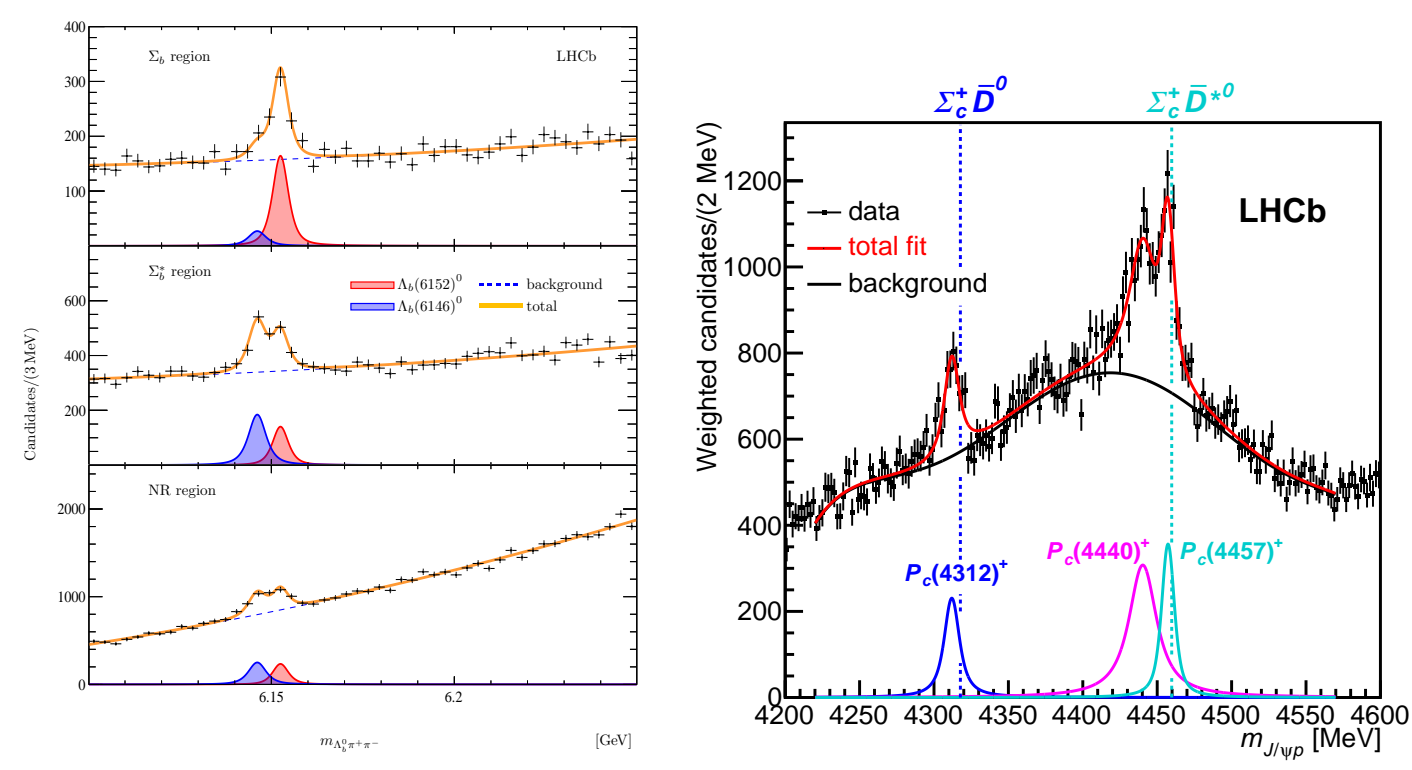

Figure 5: Left: Mass distributions of selected $\Lambda_{b}^{0} \pi^{+} \pi^{-}$candidates for the three regions in $\Lambda_{b}^{0} \pi^{+} \pi^{-}$mass: (top) $\Sigma_{b}$, (middle) $\Sigma_{b}^{*}$ and (bottom) non resonant (NR) region (from [18]). Right: Fit to the $m_{J / \psi p}$ distribution with three Breit-Wigner amplitudes for the signals and a sixth-order polynomial background. The mass thresholds for the $\Sigma_{c}^{+} D^{0}$ and $\Sigma_{c}^{+} D^{* 0}$ final states are superimposed (from [19]).

with $7.3 \sigma$ significance. The minimal quark content of these states is $d u u c \bar{c}$. Since all three states are narrow and below the $\Sigma_{c}^{+} D^{0}$ and $\Sigma_{c}^{+} D^{* 0}([d u c][u \bar{c}])$ thresholds, they provide the strongest experimental evidence to date for the existence of bound states of a baryon and a meson. These $m_{J / \psi p}$ fits alone cannot distinguish broad $P_{c}^{+}$states from other contributions that vary slowly with $m_{J / \psi p}$. Hence, a study of the $P_{c}(4380)^{+}$state observed in [20] awaits completion of an amplitude analysis.

\section{Heavy Ion physics}

\section{1 b-hadron production in proton-lead collisions}

The production of $B^{+}, B^{0}$ and $\Lambda_{b}^{0}$ hadrons is studied in proton-lead collisions at a centre-of-mass energy per nucleon pair of $\sqrt{s_{N N}}=8.16 \mathrm{TeV}$ with the proton beam pointing to the LHCb detector (forward) as well as the lead-beam in the direction of the LHCb detector (backward) [21]. Double-differential cross-sections are measured and used to determine forward-backward ratios and nuclear modification factors, which are a direct probe of nuclear effects in the production of beauty hadrons. In order to study hadronization in proton-lead collisions, ratios of $B^{0}$ over $B^{+}$and $\Lambda_{b}^{0}$ over $B^{0}$ production cross-sections are measured. Their $p_{T}$ dependence is shown in Fig. 6 (left). The ratio between meson species is consistent with being independent of rapidity and $p_{T}$ of the beauty hadrons while there is an indication for a $p_{T}$ dependence of the baryon-to-meson ratio. The baryon-to-meson cross-section ratio in proton-lead collisions is found to be compatible with the equivalent ratio measured in $p p$ collisions, and more data will be needed to study whether nuclear effects modify beauty baryon and meson production differently. 

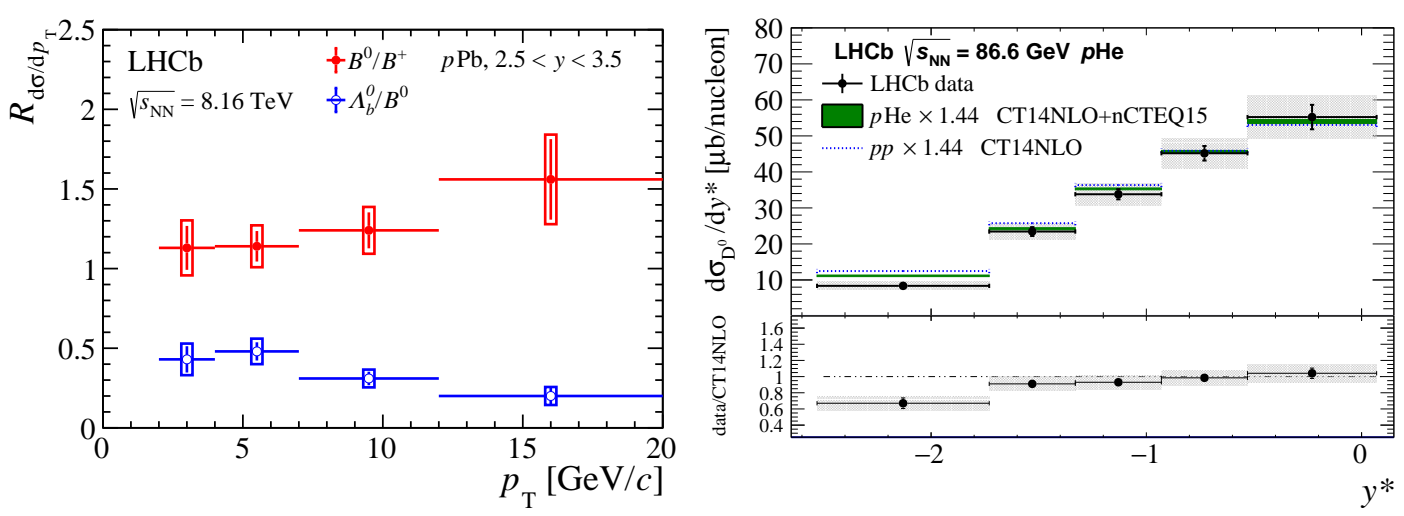

Figure 6: Left: Production cross-section ratios of $\Lambda_{b}^{0}$ over $B^{0}$ (blue) and of $B^{0}$ over $B_{s}^{0}$ (red) as a function of $p_{T}$ for $2.5<y<3.5$ (from [21]). Right: Differential $D^{0}$ production cross section for $p H e$ collisions, as a function of center-of-mass rapidity $y^{*}$. The quadratic sum of statistical and uncorrelated systematic uncertainties are indicated by the vertical black lines. The correlated systematic uncertainties are indicated by the gray area. The lower panel shows the ratio of data to predictions for $p p$ collisions (from [22]).

\subsection{Charm production in fixed target collisions}

The system for measuring overlap with gas (SMOG) device enables the injection of gases with pressure of $\mathscr{O}\left(10^{-7}\right)$ mbar in the beam pipe close to the interaction region and allows LHCb to operate as a fixed-target experiment. LHCb performed a first measurement of charm production $\left(D^{0}\right.$ and $\left.J / \psi\right)$ in fixed target collisions of protons with gaseous targets of helium and argon with nucleon-nucleon centre-of-mass energies of $\sqrt{s_{N N}}=86.6$ and $110.4 \mathrm{GeV}$, respectively [22]. The LHCb acceptance gives access to the large Bjorken- $x$ region of the target nucleon (up to $x \simeq 0.37$ for $D^{0}$ mesons), a region that might be sensitive to contributions from intrinsic charm [23]. Figure 6 (right) shows the differential $D^{0}$ cross-section as a function of the centre-of-mass rapidity. It compares very well with the shape of the differential $p p$ cross-section indicating that there is no evidence for a contribution of a valence-like charm at large rapidities.

\section{Outlook}

The LHCb experiment has collected data with a total integrated luminosity of $9 \mathrm{fb}^{-1}$ by the end of Run 2. The detector is presently being upgraded in order to improve the data taking capabilities, such as the instantaneous luminosity and the trigger efficiency. In the next running periods LHCb is expected to collect a total integrated luminosity of around $23 \mathrm{fb}^{-1}$ (end of Run 3, 2023) and $50 \mathrm{fb}^{-1}$ (end of Run 4, 2029)[24, 25]. Many of the results presented in these proceedings are statistically limited and large improvements in precision are expected with the data to be collected in Run 3 and 4. The CKM angle $\gamma$ is now measured to be $\gamma=\left(74.0_{-5.8}^{+5.0}\right)^{\circ}$ using a variety of decay channels and analysis methods and is the best determination from a single experiment. The current precision is expected to improve to $4^{\circ}$ once the full Run 2 data sample is analysed and to $1^{\circ}$ after the upgrade. $C P$ violation in charm is established by now and with the improvements in triggering and performance foreseen with the $\mathrm{LHCb}$ upgrade, future measurements are expected to have a strong 
impact on the precision of the $C P$ measurements in the charm sector. Intriguing discrepancies from the SM are observed in several measurements of semileptonic $B$ decays to leptons in the context of tests of lepton flavour universality. Several analyses are ongoing exploiting different decay channels and the full dataset currently available. Future precision measurement of exotic states at LHCb will help to shed light on the nature of the observed pentaquark and tetraquark states. Finally, there are many more results to come from special runs in Run 2 in heavy-ion and fixed-target (eg proton-neon and lead-neon) collisions. The upgrade for SMOG is ongoing and will significantly expand the fixed-target programme of $\mathrm{LHCb}$.

\section{References}

[1] A. A. Alves, Jr. et al. [LHCb Collaboration], JINST 3 (2008) S08005.

[2] R. Aaij et al. [LHCb Collaboration], Phys. Rev. Lett. 122 (2019) no.21, 211803.

[3] Heavy Flavor Averaging Group, Y. Amhis et al., Eur. Phys. J. C77 (2017) 895, updated results and plots available at https://hflav.web.cern.ch.

[4] R. Aaij et al. [LHCb Collaboration], JHEP 1407 (2014) 041;

R. Aaij et al. [LHCb Collaboration], Phys. Rev. Lett. 116 (2016) no.19, 191601.

[5] A. Cerri et al., arXiv:1812.07638 [hep-ph].

[6] LHCb collaboration,LHCb-CONF-2019-001.

[7] R. Aaij et al. [LHCb Collaboration], Phys. Rev. Lett. 118 (2017) no.26, 261803.

[8] R. Aaij et al. [LHCb Collaboration], Phys. Rev. Lett. 122 (2019) no.23, 231802.

[9] A. Di Canto et al.,Phys. Rev. D 99 (2019) no.1, 012007.

[10] LHCb collaboration,LHCb-CONF-2018-002.

[11] R. Aaij et al. [LHCb Collaboration], Eur. Phys. J. C 79 (2019) no.8, 706.

[12] R. Aaij et al. [LHCb Collaboration], Phys. Lett. B 797 (2019) 134789.

[13] R. Aaij et al. [LHCb Collaboration], arXiv:1907.10003 [hep-ex].

[14] M. Bartsch, G. Buchalla, and C. Kraus, arXiv:0810.0249;

H.-Y. Cheng and C.-K. Chua, Phys. Rev. D80 (2009) 114026.

[15] R. Aaij et al. [LHCb Collaboration], Phys. Rev. Lett. 122 (2019) no.19, 191801.

[16] R. Aaij et al. [LHCb Collaboration], arXiv:1905.06614 [hep-ex].

[17] R. Aaij et al. [LHCb Collaboration], arXiv:1909.01010 [hep-ex].

[18] R. Aaij et al. [LHCb Collaboration], Phys. Rev. Lett. 123 (2019) 152001.

[19] R. Aaij et al. [LHCb Collaboration], Phys. Rev. Lett. 122 (2019) no.22, 222001.

[20] R. Aaij et al. [LHCb Collaboration], Phys. Rev. D 94 (2016) no.7, 072001.

[21] R. Aaij et al. [LHCb Collaboration], Phys. Rev. D 99 (2019) no.5, 052011.

[22] R. Aaij et al. [LHCb Collaboration], Phys. Rev. Lett. 122 (2019) no.13, 132002.

[23] J. Pumplin, H. L. Lai, and W. K. Tung, Phys. Rev. D 75, 054029 (2007);

S. Dulat et al., Phys. Rev. D 89, 073004 (2014).

[24] R. Aaij et al. [LHCb Collaboration], Eur. Phys. J. C 73 (2013) 2373.

[25] I. Bediaga et al. [LHCb Collaboration], arXiv:1808.08865, LHCB-PUB-2018-009. 